\title{
La profesionalización en la empresa familiar y su influencia en el proceso de sucesión: análisis preliminar
}

\author{
Arnoldo Edoardo Ortiz Vargas ${ }^{1}$ \\ Ma. Carmen Liquidano Rodríguez ${ }^{2}$ \\ María de los Ángeles Silva Olvera ${ }^{3}$
}

\begin{abstract}
Resumen
En este estudio de investigación se presentan los resultados preliminares de la influencia de la profesionalización en el proceso de sucesión de las Empresas Familiares (EF) en Aguascalientes. La metodología de investigación de este estudio fue cuantitativo, no experimental y bivariado. $\mathrm{Y}$ Se desarrolló un cuestionario semiestructurado aplicado satisfactoriamente a 18 empresas familiares. Los resultados de este estudio fueron obtenidos mediante un análisis de correlación con la prueba de Rho de Spearman con un resultado de 0.630, lo que demuestra que existe una fuerte influencia entre las variables.
\end{abstract}

Palabras clave: Profesionalización, empresa familiar, sucesión.

\section{Abstract}

This study presents the preliminary results of the influence of professionalization on the succession process of family business (FB) in Aguascalientes. The study research methodology was quantitative, non-experimental, and bivariate. A semi-structured questionnaire was successfully applied to 18 family companies. The results of this study were obtained through a correlation analysis with the Spearman Rho test with a result of 0.630 , which shows that there is a strong influence between the variables.

Keywords: Professionalization, family business, Succession.

\footnotetext{
${ }^{1}$ Estudiante de la maestría en ciencias en administración del Instituto Tecnológico de Aguascalientes.

2 Docente - investigadora del Instituto Tecnológico de Aguascalientes. Miembro del CAC Gestión y Desarrollo de Empresas ITAGU-CA-3, y perfil PRODEP vigente.

${ }_{3}^{3}$ Docente - investigadora del Instituto Tecnológico de Aguascalientes. Miembro del CAC Gestión y Desarrollo de Empresas ITAGU-CA-3, perfil PRODEP vigente y presidenta del Consejo de Posgrado.
} 


\section{Introducción}

Tres son los retos principales que enfrentan las empresas familiares: la planificación de la sucesión en la dirección, la profesionalización y las posibilidades de supervivencia (Zúñiga-Vicente y Sacristán-Navarro, 2009). El autor Tadeo-Basco (2005), en sus resultados argumentan que las empresas familiares (EF) integradas tuvieron mejores índices de eficiencia que las no familiares, e identificaron la existencia de relación entre la forma en que las empresas combinan las prácticas de gestión y gobierno y el tipo de relación que se genera en la familia.

Es importante destacar que de acuerdo a GómezBetancourt et al (2012), la EF en Aguascalientes, genera beneficios como la participación en el crecimiento del producto interno bruto (PIB), la conservación de empleos, empleadores más preparados con nuevas visiones, así como ser una tradición de consumo en el ámbito comercial, con valores muy arraigados, lo que se fortalece con la información del INEGI (2009), de que, en el ámbito nacional mexicano, genera oportunidades de empleo y contribuye en los indicadores macroeconómicos del país. (López-Parra, M.E. et al., 2015). Soto (2013) destaca que la investigación sobre la sucesión de las EF en México, no tiene el auge que debería tener y su difusión carece de espacios especializados, además señala que, después de la transición generacional, la supervivencia de la EF es baja.

En la presente investigación se derivan las siguientes hipótesis relacionadas con la profesionalización, el proceso de sucesión y la empresa familiar.

HI: La profesionalización de la empresa familiar influye en el proceso de sucesión.

Ho: La profesionalización de la empresa familiar no influye en el proceso de sucesión.

El objetivo general de esta investigación fue analizar la relación de la profesionalización de la empresa familiar con el proceso de sucesión, en Aguascalientes.

Revisión literaria.

\section{La profesionalización}

Cuando el control directivo de la empresa familiar se transfiere a personal externo a la familia, o cuando la EF adopta profesionales para gestionar mejor la complejidad empresarial enfrentándose al ambiente interno y externo, se le conoce como profesionalización. (Robichaud, 2009). La profesionalización es independiente de la pertenencia a la propiedad familiar (Meroño-Cerdán, 2009), para Tadeo-Basco (2005), la profesionalización es sinónimo de dirección profesional, esto "es la capacidad interna con que una empresa familiar estructura, gestiona y gobierna los recursos y capacidades que le son propios de tal forma que puede conseguir resultados deseados para el logro de sus objetivos" (p. 100). De preferencia debe darse cuando la EF haya alcanzado un nivel de madurez óptimo o cuando exista la ausencia de un sucesor que sea capaz de dirigir o este calificado para la gestión de la empresa familiar, Robichaud (2009). Para Meroño-Cerdán (2009), es el estudio de la figura del gerente y la estructura organizacional, haciendo énfasis es la importancia de estudiar al gerente gestionante de la EF, en virtud de que centralizan sus funciones y toma de decisiones.

\section{La sucesión}

La sucesión se ha definido como el relevo en la dirección o el liderazgo de la empresa del propietariofundador al sucesor, que suele ser un miembro de la misma familia (Zúñiga-Vicente y Sacristán-Navarro, 2009). Este proceso integra la creación de documentos que organicen la transferencia de la propiedad de la firma, desde el dueño hacia otros (familiares, directivos ajenos a la familia, asociaciones de caridad, etc.) de la manera más eficiente desde el punto de vista propositivo. (Arenas-Cardona y Rico-Balvín, 2014). Según Arreola-Bravo, et al (2015), a través de la sucesión se conserva generacionalmente aquello que simboliza la tradición y jerarquía de la familia donde se involucra a los miembros de la misma, con una visión a largo plazo de una inversión que será heredada a sus descendientes futuros. Para Martínez-Echezárraga (2012), es la transmisión del mando y de la propiedad de una generación a otra. 
El proceso sucesorio implica "la aparición de nuevos líderes a veces tienen patrones simbólicos, creencias y valores diferentes a las que hasta entonces entraban en vigor en la organización" (Estol y Ferreira (2006)).

\section{Empresa familiar}

Una empresa familiar es aquella empresa cuyo control está en manos de más de dos miembros familiares (Gallo, 1998). Sean directos o indirectos de una misma línea genealógica, que trabajan en conjunto con valores y visiones los cuales son afectados por los niveles culturales que cada una de estas familias presenta de forma única en cada organización. Rojas-Roldan (2012) dice que una empresa familiar es aquella organización de carácter económico cuyo objeto principal sea la producción o comercialización de bienes y servicios, y cuya propiedad pertenece, en su totalidad o en una mayoría, a un grupo de personas unidas por un vínculo familiar.

\section{Metodología de investigación.}

Se diseñó un estudio no experimental, de corte cuantitativo, exploratorio, descriptivo y correlacional, teniendo como unidad de análisis a las empresas familiares de Aguascalientes, con un muestreo no probabilístico, definiendo como criterios de selección de la muestra, a empresas familiares que hayan realizado una sucesión, no importando si es segunda o tercera generación, en el entendido que los resultados no podrán ser generalizables, sino únicamente aplicables a la muestra de estudio. Se diseñó un cuestionario, obteniendo la confiabilidad con el método Alpha de Cronbach, obteniendo valores superiores al .690, hasta el momento en este análisis preliminar, se ha obtenido una muestra de 18 empresas familiares, a través de contactos y dueños de negocio.

El cuestionario, se adaptó para aplicarse a través de Internet mediante la plataforma Google Forms, el cual no se aplicó para esta información. En la medición de la profesionalización se utilizaron las dimensiones: perfil del tomador de decisiones, estructura organizacional, tecnología de la información, planeación estratégica y prácticas de recursos humanos.
Y en el proceso de sucesión, las dimensiones fueron: planeación de la sucesión en la empresa, el sucesor de la empresa familiar, desarrollo de la organización, desarrollo de las relaciones familiares.

\section{Resultados}

Entre los principales resultados, las EF de esta muestra pertenecen el $44.4 \%$ al sector servicios, el $33.3 \%$ a manufactura y el $22.2 \%$ al comercio. En esta muestra el que dirige la administración, es la generación fundadora con $61.1 \%$. Figura 1.

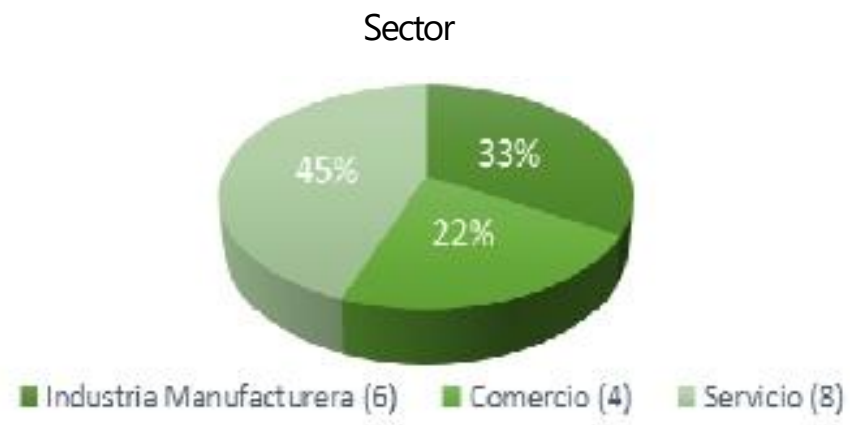

Figura 1 Sector

Fuente: Elaboración propia (2016)

En cuanto al tamaño de la empresa, predomina la microempresa con ventas anuales de 0 a 4 millones de pesos, donde las decisiones son tomadas por el fundador, quien es predominantemente masculino $(77.8 \%)$, con escolaridad de licenciatura (38.9\%). El $100 \%$ de los integrantes de esta muestra son familiares. Figura 2.

Ventas anuales
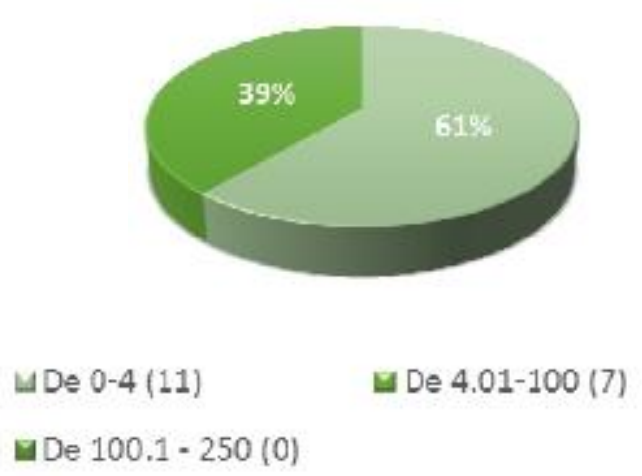

Figura 2 Clasificación de empresas Fuente: Elaboración propia (2016) 
Estructura Organizacional

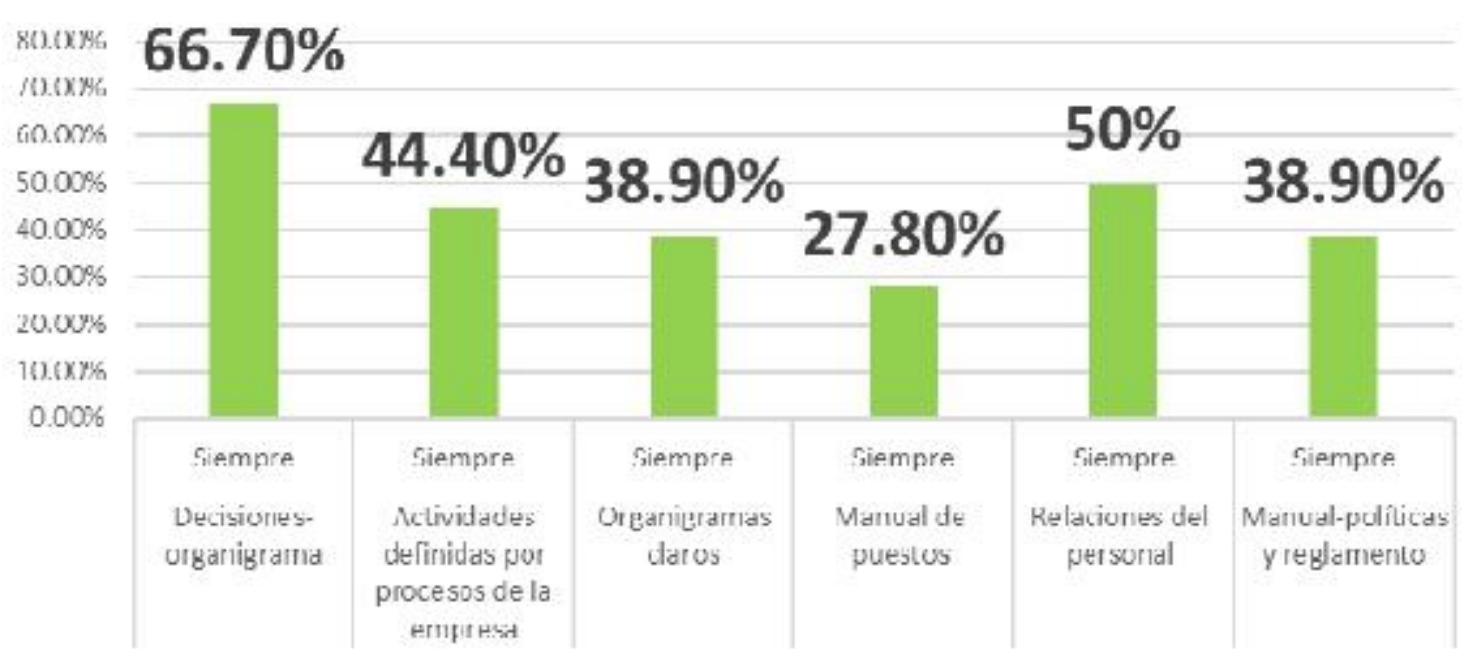

Figura 4 Estructura organizacional de las EF

Desarrollo de la Organización

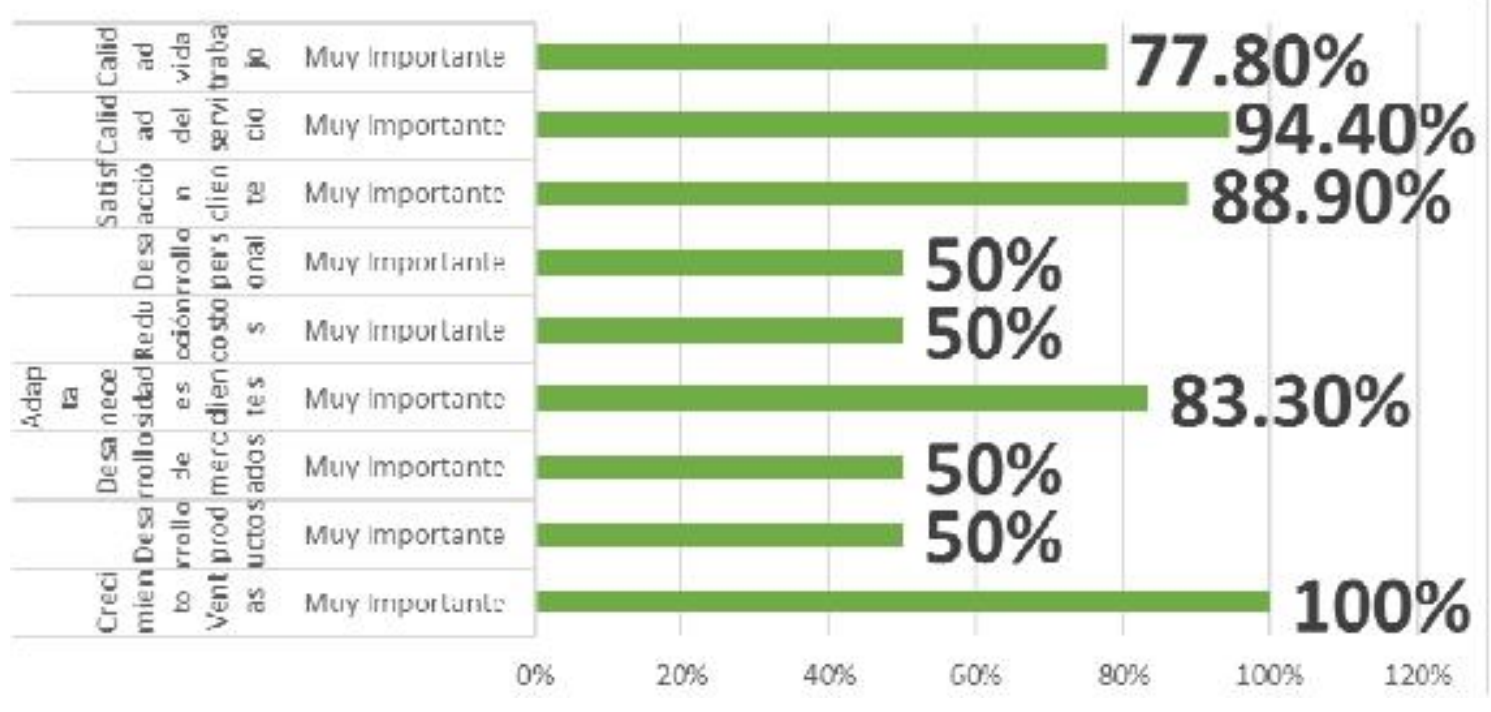

Figura 5 Desarrollo de las empresas familiares 
Las empresas familiares arrojan resultados negativos en cuanto a su próxima sucesión, y no cuentan con un plan de sucesión por escrito, en su mayoría hay un 94\% que no lo ha planeado, y solo un $6 \%$ si tiene un plan de sucesión para la siguiente generación, según se muestra en la figura 3.

Figura 3. Plan de sucesión por escrito

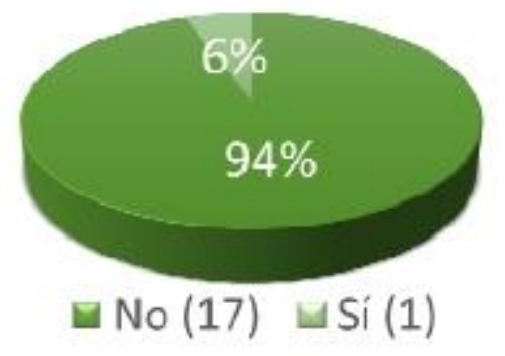

Fuente: Elaboración propia (2016)

Los resultados obtenidos mediante el sistema SPSS fueron de 0.630 , lo cual nos indica que existe una correlación positiva, aceptando así:

HI: La profesionalización de la empresa familiar influye en el proceso de sucesión.

\section{Conclusiones}

Se cuenta con evidencia empírica de la variable profesionalización y su influencia en el proceso de sucesión en empresas familiares; sin embargo aún no es representativa de la población de estudio. En estos resultados preliminares fue mayor la participación de EF con ventas menores de 4 millones anuales, predominante las empresas de servicio, administradas por la generación fundadora $y$ en importancia la $2^{a}$. Generación, pero que no cuentan con plan de sucesión por escrito, solo prácticas que se pasan verbalmente.

Además predominan decisiones con base en la jerarquía del organigrama, y las relaciones del personal entre los departamentos; sin embargo, se observa que es relativamente bajo que las empresas cuenten con descripciones de puestos. Destaca que no utilizan el intranet para comunicación interna, sin embargo si realizan actividades laborales a través de internet.

Pocas veces los directivos participan en capacitación, se les brinda capacitación a nuevos directivos, se da capacitación para socializar con la cultura de la empresa, evalúan el desempeño por resultados.

Tabla 4.1 Resultados de correlación.

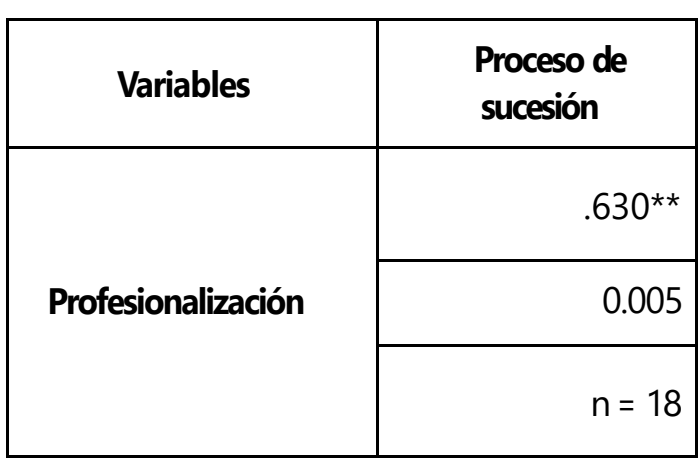

Fuente: Elaboración propia (2016)

La estructura organizacional delas empresas familiares muestra además que el $66.70 \%$ toman sus decisiones en base al organigrama de la empresa. El $38.90 \%$ de la empresa considera que estos organigramas son aplicados y definidos en forma clara. Aunado a esto, se entiende que el $44.40 \%$ de estas $E F$, siempre siguen sus actividades definidas y apegados a los procesos de la empresa, y solo el $27.80 \%$ de estos aplica el manual de descripción de políticas y reglamentos en la empresa familiar, así como se muestra en la figura 4.

El desarrollo de la organización en la empresa familiar muestra que las empresas familiares en su totalidad del $100 \%$ se orientan y da prioridad al crecimiento de sus ventas. Cabe destacar que las EF comparten esta necesidad de ofrecer un servicio de calidad en el manejo de sus negocios, obteniendo así el $94.40 \%$ tal y solo el $88.90 \%$ está orientada a buscar la satisfacción del cliente, como se muestra en la figura 5.

Para probar la hipótesis estadística, se utilizó la prueba de correlación con Rho de Spearman, obteniendo los siguientes resultados en la tabla 1. 
Existe mayor correlación de las prácticas de recursos humanos en el proceso de sucesión, sin embargo en esta muestra preliminar aún no se cuenta con el $50 \%$ o más de los datos. Se obtuvo evidencia empírica fuerte correlación de la estructura orgánica en el proceso de sucesión en cuanto al desarrollo organizacional.

Limitantes de estudio

La principal limitante de la investigación fue por falta de la apertura para responder o el poco interés en la investigación, las razones se desconocen del porqué no hay apertura por parte de las EF.

\section{Referencias}

Arenas-Cardona, H., Rico-Balvín, D. (2014). La empresa familiar, el protocolo y la sucesión familiar. Estudios gerenciales., vol.30, n.132, pp. 252-258. ISSN 0123-5923.

ttp://dx.doi.org/10.1016/j.estger.2014.02.013

Arreola-Bravo, F. M., Niebla-Zatarain, J. C.; RamírezMartínez, G. (2015). Los factores socioemocionales y el proceso de sucesión en las empresas familiares Ciencia Ergo Sum, vol. 22, núm. 2, julio-octubre, 2015, pp. 103115 Toluca, México

http://www.redalyc.org/articulo.oa?id=10439327001ER

Cronbach, L. J. (1951). "Coefficient alpha and the internal structure of tests». Psychometrika vol. $16 \mathrm{pp}$ 297-334. Doi: 10.1007/BF02310555. ISSN 0033-3123

Gallo, M. (1998). La sucesión en la empresa familiar. Barcelona, España. Editorial La Caixa.

Gómez- Betancourt, G., López-Vergara, M. P. y Betancourt-Ramírez, J. B. (2008). Estudio exploratorio de los factores que influyen en la selección de un mecanismo de sucesión patrimonial en las empresas familiares colombianas, Cuadernos de administración, pp 269-292, julio-diciembre,

http://mww.redalyc.org/articulo.oa?id=20503712> ISSN 0120-3592
Felipe-Estol, M. y Ferreira, M. (2006). O processo sucessório e a cultura organizacional em uma empresa familiar brasileira. Revista de Administração

Contemporânea. 2006, vol.10, n.4, pp. 93-110. ISSN 1982-7849.

\section{http://dx.doi.org/10.1590/S1415-65552006000400005}

INSTITUTO NACIONAL DE ESTADISTICA Y GEOGRAFIA. (2009) Micro, pequeña, mediana y gran empresa; estratificación de los establecimientos. Censos económicos 2009.

http://www.inegi.org.mx/est/contenidos/espanol/proye ctos/censos/ce2009/pdf/Mono_Micro_peque_mediana.p df

López-Parra, M. E., González-Navarro, N. E. y Vargas-Marín, M. E. (2015). Empresas familiares e innovación, un diagnostico en la micro y pequeña empresa de ciudad Obregón, Sonora, Revista Ciencias Administrativas, Teoría y Praxis. pp (55-72) Baja California, México.

Martínez-Echezárraga, J. (2012). Empresas familiares: reto al destino. Buenos Aires, Argentina. Editoriales Granica, SA.

Meroño-Cerdan, A. L. (2009) Análisis del nivel de profesionalización de la empresa familiar. Revista de Estudios Empresariales. Segunda época. Número: 2.p.p (80 - 98). Murcia, España.

http://revistaselectronicas.ujaen.es/index.php/REE/arti cle/view/350

Robichaud, D. (2009). La professionnalisation des PME familiales: une voie privilégiée pour la relève dans les organisations. Revue économique et sociale: bulletin de la Société d'Etudes Economiques et Sociales p.p (95108) http://dx.doi.org/10.5169/seals-142303

Rojas-Roldan, J. (2012) La profesionalización de las pequeñas y medianas empresas familiares de servicios del municipio de Chalco, estado de México, como factor de éxito para su continuidad. Instituto Politécnico Nacional. México, Ciudad de México. 
Soto, A., (2013). La empresa familiar en México: Situación actual de la investigación. Contaduría y Administración, abril-junio 2013: pp 135-171. http://www.elsevier.es/es/linksolver/unam/unampii/retriev e/pii/S0186104213712130?_returnURL=http\%3A\%2F\%2 Flinkinghub.elsevier.com\%2Fretrieve\%2Fpii\%2FS01861 04213712130\%3Fshowall\%3Dtrue

Tadeo-Basco, J. R. (2005). Comportamientos en la dirección y gobierno de la empresa familiar: análisis empírico de la profesionalización como garantía de continuidad. Madrid, España.

Zúñiga-Vicente, J.A., Sacristán- Navarro, M. (2009). Los directivos externos y la sucesión en la empresa familiar: un caso de estudio Universia Business Review, núm. 22, pp. 74-87. Madrid, España http://www.redalyc.org/articulo.oa?id=43311704005ER 